\title{
Polibotánica
}

ISSN electrónico: 2395-9525

POLIBETÁNICA

polibotanica@gmail.com

Instituto Politécnico Nacional

México

http:www.polibotanica.mx

\section{DIVERSIDAD, ESTRUCTURA ARBÓREA E ÍNDICE DE VALOR DE IMPORTANCIA EN UN BOSQUE URBANO DE LA CIUDAD DE MÉXICO}

\section{DIVERSITY, TREE STRUCTURE, AND IMPORTANCE VALUE INDEX IN AN URBAN PARK IN MEXICO CITY}

Saavedra-Romero, L. de L.; P. Hernández-de la Rosa, D. Alvarado-Rosales, T. MartínezTrinidad, y J. Villa-Castillo.

DIVERSIDAD, ESTRUCTURA ARBÓREA E ÍNDICE DE VALOR DE IMPORTANCIA EN UN BOSQUE URBANO DE LA CIUDAD DE MÉXICO.

DIVERSITY, TREE STRUCTURE, AND IMPORTANCE VALUE INDEX IN AN URBAN PARK IN MEXICO CITY.

POLIBeTÁNICA

Instituto Politécnico Nacional
Núm. 47: 25-37 México. Enero 2019

DOI: $10.18387 /$ polibotanica.47.3 


\section{DIVERSIDAD, ESTRUCTURA ARBÓREA E ÍNDICE DE VALOR DE IMPORTANCIA EN UN BOSQUE URBANO DE LA CIUDAD DE MÉXICO}

\section{DIVERSITY, TREE STRUCTURE, AND IMPORTANCE VALUE INDEX IN AN URBAN PARK IN MEXICO CITY}

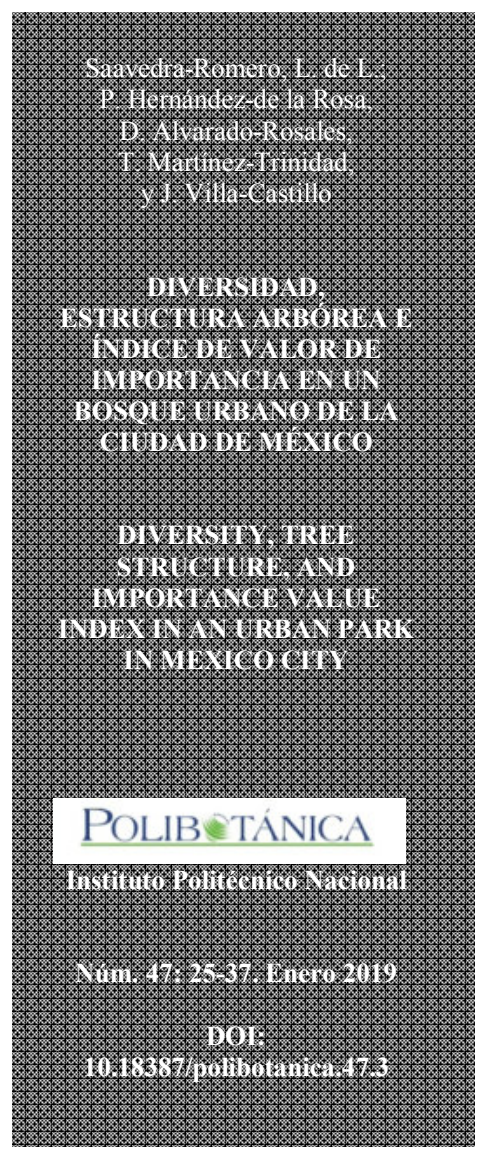

\author{
L. de L. Saavedra-Romero \\ Postgrado en Fitosanidad-Fitopatología. \\ Colegio de Postgraduados, Campus Montecillo. Km. 36.5 \\ Carretera México-Texcoco, Montecillo Texcoco, Estado de México, CP 56230. \\ P. Hernández-de la Rosa / pathr@colpos.mx \\ Postgrado en Ciencias Forestales. \\ Colegio de Postgraduados, Campus Montecillo. Km. 36.5 \\ Carretera México-Texcoco, Montecillo Texcoco, Estado de México, CP 56230. \\ D. Alvarado-Rosales \\ Postgrado en Fitosanidad-Fitopatología. \\ Colegio de Postgraduados, Campus Montecillo. Km. 36.5 \\ Carretera México-Texcoco, Montecillo Texcoco, Estado de México, CP 56230. \\ T. Martínez-Trinidad \\ Postgrado en Ciencias Forestales. \\ Colegio de Postgraduados, Campus Montecillo. Km. 36.5 \\ Carretera México-Texcoco, Montecillo Texcoco, Estado de México, CP 56230. \\ J. Villa-Castillo \\ Comisión Nacional Forestal (CONAFOR) \\ Sede Guadalajara. Zapopan, Jalisco, México.
}

RESUMEN: El arbolado urbano es un elemento esencial en la infraestructura de toda urbe. El Bosque San Juan de Aragón (BSJA) en la Ciudad de México está catalogado como un área verde de valor ambiental pero su condición respecto a la diversidad y estructura arbórea se ha estudiado poco. El propósito del presente trabajo fue aplicar los procedimientos del Indicador Diversidad y Estructura, en el área verde urbana, Bosque San Juan de Aragón de la Ciudad de México, y contribuir en el diagnóstico sobre el estado actual de salud en esta materia.

Se ubicaron al azar 28 sitios de muestreo circulares de 0.1 ha; en cada uno se determinó por árbol, la clasificación taxonómica, el diámetro normal (Dn), la altura total (At) y diámetro de copa. En gabinete se calculó el área basal (Aba), volumen compuesto y área superficial de copa y los índices de diversidad de Simpson, Berger-Parker, además, de un índice de valor de importancia urbano (IVIU). Se identificaron 10 familias, 11 géneros y 12 especies. Las familias Casuarinaceae, Proteaceae, Myrtaceae y Cupressaceae estuvieron representadas en un 69\%. El Dn promedio fue de $17.5 \mathrm{~cm}$, la At de $8.6 \mathrm{~m}$ y el Aba de $0.40 \mathrm{~m}^{2} / \mathrm{ha}$. El valor para el índice de Simpson fue de 0.85 que indica una alta dominancia y baja riqueza de especies y el de Berger-Parker fue de 0.66 , concentrado la abundancia en cuatro especies. Casuarina equisetifolia y Eucalyptus camaldulensis presentaron los IVIU más altos con $31.98 \%$ y $19.96 \%$, respectivamente. La diversidad arbórea del bosque fue baja, similar a otras áreas verdes urbanas. Por medio del IVIU se jerarquizó la importancia por especie, y se identificó a 
las de mayor potencial actual para proveer servicios ecosistémicos debido a sus altos porcentajes de biomasa y cobertura.

Palabras clave: bosque urbano, salud forestal, índices de diversidad.

\begin{abstract}
Urban trees are an essential component of all city's facilities. The San Juan de Aragon Park (SJAP) in Mexico City, is considered a green area with environmental value; however, there is little research on the diversity and structure as a health indicator. The aims of the study were to adapt the Forest Health methodology for tree diversity and structure indicators (DSI) to assess SJAP current's condition. A total of 28 circular sites ( 0.1 ha) were randomly established. For each tree, taxonomic identification, normal diameter (Nd) and total height (Th) were registered. Basal area $(\mathrm{Ba})$, compound volume, crown surface as well as the Simpson, Berger-Parker, and an urban importance value index (UIVI) was determined. The Casuarinaceae, Proteaceae, Myrtaceae and Cupressaceae families were the most abundant with $69 \%$ of the total density. Average Nd, Th and Ba were $155 \mathrm{~cm}, 8.6 \mathrm{~m}$, and $0.40 \mathrm{~m}^{2} \mathrm{ha}^{-1}$, respectively. Simpson index was 0.85 representing a high dominance y low richness, BergerParker values (0.66) concentrates abundance in four species. The highest UIVI were obtained for Casuarina equisetifolia (31.98\%), and Eucalyptus camaldulensis (19.96\%). Tree diversity was low, similar to other urban green areas. The UIVI identifies species with the actual highest potential to provide ecosystem services due to their highest biomass and coverage.
\end{abstract}

Key words: urban forest, forest health, diversity indexes.

\title{
INTRODUCCIÓN
}

La determinación de la condición actual de las áreas verdes urbanas se ha extendido a varios tópicos, pero el estudio de su diversidad, distribución y estructura son los de mayor impacto (Baró et al., 2014; Burkhart \& Tomé, 2012; Savard, Clergeau, \& Mennechez, 2000). Los beneficios que aportan los árboles, están directamente relacionados con su condición actual de salud (Saavedra-Romero et al., 2016), sus dimensiones (Li, Wang, \& Huang, 2011; Troxel, Piana, Ashton, \& Murphy-Dunning, 2013), y cobertura (Mori, Hanslin, Burchi, \& Sæbø, 2015; Schomaker et al., 2007), en este sentido, su valor ecológico-ambiental se incrementa cuando su eficiencia en la captura de contaminantes es alta. Su capacidad de interceptar y absorber contaminantes depende de la especie arbórea en cuestión, y del volumen de sus copas (Korhonen, Vauhkonen, Virolainen, Hovi, \& Korpela, 2013), del área superficial e índice de área foliar, de su topografía foliar (Janhäll, 2015) y, por supuesto, del grupo arbóreo en cuestión (angiosperma o gimnosperma) (Beckett, Freer-Smith, \& Taylor, 2000; Räsänen et al., 2013). Por ejemplo, árboles sanos de más de $75 \mathrm{~cm}$ de diámetro eliminan 60 a 70 veces más contaminantes atmosféricos al año $(1.4 \mathrm{~kg})$ que los árboles pequeños menores a $7 \mathrm{~cm}$ de diámetro $(0.025 \mathrm{~kg})$ (Nowak \& Heisler, 2010).

En la actualidad, el Programa de Análisis e Inventario de Bosques (FIA) de Estados Unidos, emplea un conjunto de indicadores de salud forestal los cuales se diseñaron para determinar la condición actual de sus ecosistemas forestales, identificar cambios y detectar tendencias a largo plazo (Woodall et al., 2011), entre ellos: (a) la condición de copa (Saavedra-Romero et al., 2016; Randolph, Morin, \& Steinman, 2010); (b) daños al arbolado (Zaragoza-Hernández et al., 2015), y (c) diversidad y estructura de la vegetación (Schulz, Bechtold, \& Zarnoch, 2009), y pese a que fueron diseñados para el estudio de bosques naturales, en años recientes aplican sus atributos también al estudio del bosque urbano (Buckelew, Twardus, \& Nowak, 2008). En lo que respecta al indicador diversidad y estructura, sus esfuerzos son dirigidos al inventario de plantas vasculares sobre una red sistemática de conglomerados a través del territorio estadounidense (Potter, 2017), y entre los datos e información derivada de su uso, está la composición y riqueza de especies, abundancia, estructura horizontal y vertical, frecuencia de especies nativas e introducidas, así como la identificación de tasas de cambios de especies en el largo plazo (Schulz, 2017). Si bien, estos procedimientos se han reportado en diversas 
investigaciones, es preciso aplicarlos bajo condiciones particulares para extender su uso (Schroeder, Healey, \& Moisen, 2010; Schulz, Bechtold, \& Zarnoch, 2009), éste es el caso de los bosques urbanos. Por tanto, el propósito del presente trabajo fue aplicar los procedimientos del indicador diversidad y estructura en el área verde urbana, Bosque San Juan de Aragón de la Ciudad de México, y contribuir en el diagnóstico sobre el estado actual de salud en esta materia.

\section{MÉTODOS}

\section{Área de estudio}

El presente estudio se realizó en el Bosque de San Juan de Aragón (BSJA) localizado al noreste de la Ciudad de México, a una altitud promedio de 2240 m, dentro de la Alcaldía Gustavo A.

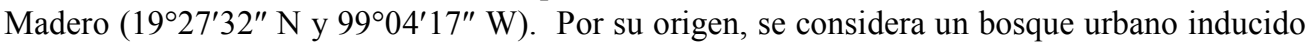
con una superficie de área verde de 114 ha dividida en 14 secciones, de las que se seleccionaron 10 al azar identificadas con los caracteres B, C, E, F, G, H, J, K, L y M (fig. 1).

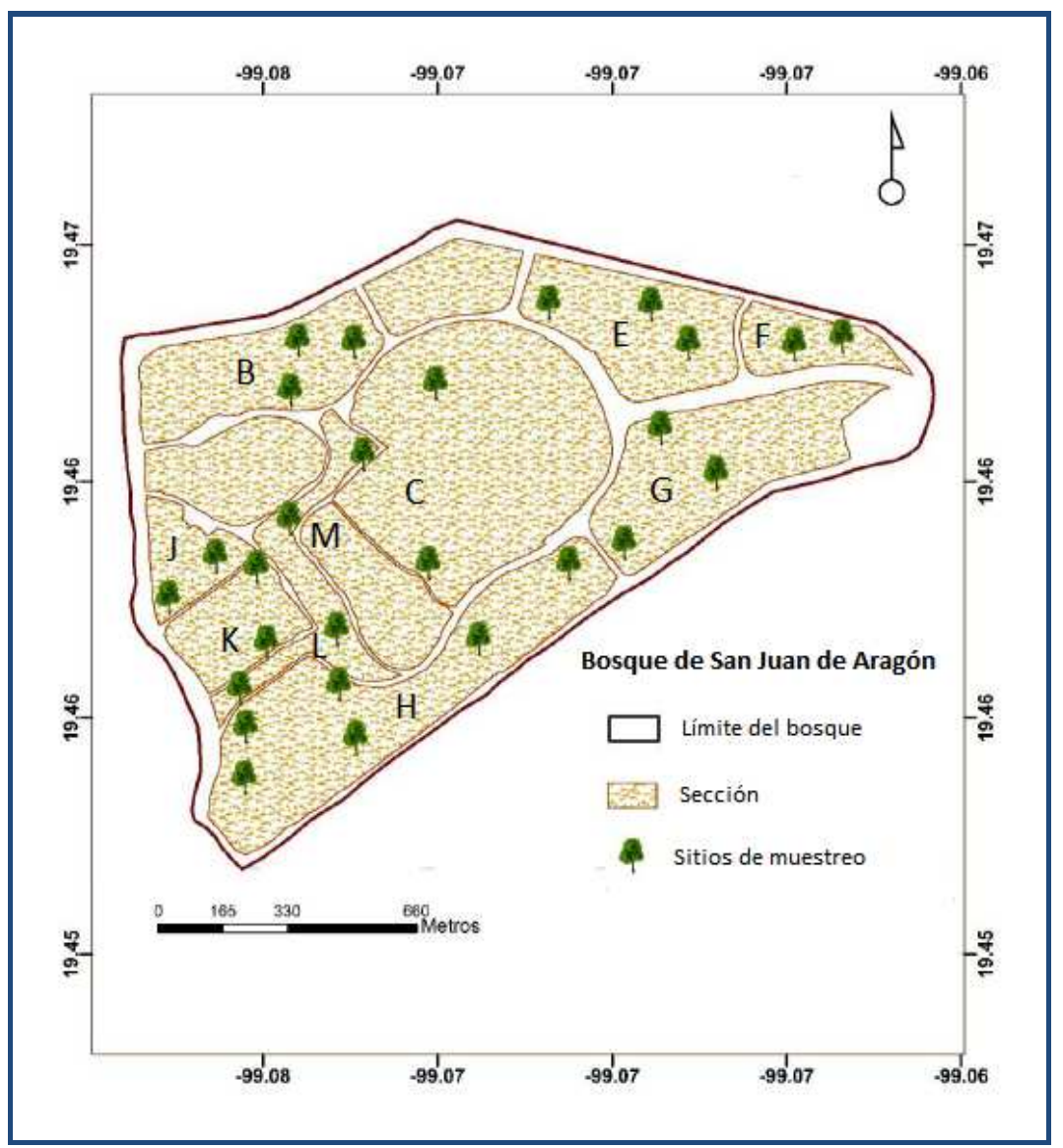

Fig. 1. Polígono del Bosque de San Juan de Aragón, Ciudad de México, secciones y sitios de muestreo.

\section{Diseño de muestreo}

De acuerdo con la tipología de áreas verdes de uso público de nuestro país, respecto a dimensiones, diseño arquitectónico, función, y espacios verdes arbolados (Flores-Xolocotzi \& González-Guillén, 2010), se emplearon sitios de muestreo circulares de dimensiones fijas. En 
este sentido, se establecieron aleatoriamente 28 unidades de muestreo de 0.1 ha, lo que representó una intensidad de muestreo del 2.5\% aceptada para áreas urbanas (Schreuder, Ernst, \& Ramírez, 2004). Cada árbol dentro de los sitios fue numerado iniciando por el norte (Schulz et al., 2009), se identificaron taxonómicamente, se midió diámetro normal (Dn) con una cinta diamétrica, diámetro de copa con flexómetro y en sentido de los puntos cardinales y altura total con una pistola Haga; en gabinete, se calculó el área basal (Aba) (Romhan-de la Vega \& Ramírez-Maldonado, 2010). El levantamiento de la información se realizó durante la estación de crecimiento junio-agosto del 2014.

\section{Índices de diversidad y valor de importancia urbano}

Se calculó el índice de diversidad de Simpson (1-D) considerado como uno de los más robustos para obtener una estimación de la diversidad, especialmente cuando se tienen muestras relativamente pequeñas (Magurran, 2004) y el índice de Berger-Parker ya que provee una medida simple y de fácil interpretación sobre la abundancia de las especies (Landeros \& Cerna, 2007), además de un índice de valor de importancia urbano (IVIU) por especie; para éste, y con el fin de obtener un índice más robusto y completo, se incluyeron cuatro variables de copa en su cálculo: (a) el volumen compuesto de copa, (b) el área superficial de copa (variables compuestas-tridimensionales) (Schomaker et al., 2007; Zarnoch, Bechtold, \& Stolte, 2004), y dos variables absolutas, (c) la densidad de copa y (d) la proporción de copa viva, recomendadas por Saavedra-Romero et al., (2016). El volumen compuesto y el área superficial fueron calculados de la siguiente forma (ecuación 1 y 2):

$$
\begin{gathered}
V c c=\left(0.5 \mathrm{mr}^{2}(\mathrm{Lc})\right)\left(\frac{\operatorname{DenC}}{100}\right) \ldots \ldots \ldots \ldots \ldots \ldots \ldots \ldots \ldots \ldots \\
\mathrm{Asc}=\frac{4 \pi \mathrm{Lc}}{3 \mathrm{r}^{2}}\left[\left(\mathrm{r}^{2}+\frac{\mathrm{r}^{4}}{4 \mathrm{~L} \mathrm{~s}^{2}}\right)^{1.5}-\left(\frac{\mathrm{r}^{4}}{4 \mathrm{~L} \mathrm{c}^{2}}\right)^{1.5}\right] \frac{\operatorname{Denc}}{100} \ldots \ldots
\end{gathered}
$$

Donde:

$$
\begin{aligned}
& \mathrm{Vcc}=\text { volumen compuesto de copa }\left(\mathrm{m}^{3}\right) \\
& \text { Asc }=\text { área superficial de copa }\left(\mathrm{m}^{2}\right) \\
& \mathrm{R}=\text { radio de copa }(\mathrm{m}) \\
& \mathrm{Lc}=\text { At }\left(\frac{\mathrm{Pc}}{100}\right)(\mathrm{m}) \\
& \text { Pcv = proporción de copa viva }(\%) \\
& \mathrm{Lc}=\text { largo de copa } \\
& \text { At }=\text { altura total del árbol }(\mathrm{m}) \\
& \text { DenC }=\text { densidad de copa }(\%) \\
& \pi=3.1416
\end{aligned}
$$

Otros parámetros incluidos en el IVIU, fueron dominancia, frecuencia y altura total. El valor final del índice por especie se obtuvo mediante de la suma de los cinco componentes incluidos en la ecuación 3:

$$
\mathrm{IVIU}=\frac{(\mathrm{Fr}+\mathrm{Dr}+\mathrm{Ar}+\mathrm{Vacr}+\mathrm{Ascr})}{\mathrm{B}}
$$

Donde:

$F_{r}=$ frecuencia relativa; $D_{r}=$ dominancia relativa; $A_{r}=$ altura relativa; $V_{c c}=$ volumen compuesto de copa relativo y $\mathrm{Asc}_{\mathrm{r}}=$ área superficial de copa relativa. 
Las ecuaciones para calcular dominancia y frecuencia relativa se obtuvieron de acuerdo a Mueller-Dombois \& Ellenberg (1974), mientras que los valores relativos para altura, volumen compuesto y área superficial de copa, se obtuvieron de la siguiente forma:

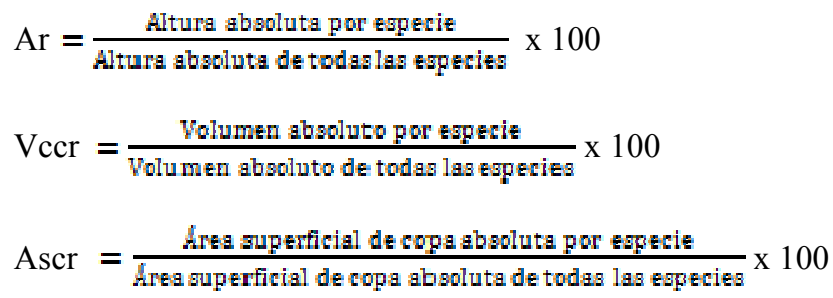

A través de este IVIU aparentemente complejo, se pretende jerarquizar la importancia de cada especie de manera horizontal al incluir la dominancia y frecuencia (García-Mayoral, ValdezHernández, Luna-Cavazos, \& López-Morgado, 2015), así como de manera vertical y tridimensional, al incluir la altura, volumen y área superficial de copa. Un índice de valor forestal con características similares fue abordado por Tellez-García \& Valdez-Hernández (2012).

\section{Análisis de datos}

Con los datos de campo, se creó una base de datos en Excel, a partir de las cuales se pudieron construir las gráficas de distribución de diámetros, alturas y cálculo de los índices; se realizaron análisis estadísticos con el programa SAS ${ }^{\circledR}$ (Statistical Analysis System) Versión 9.4. Para determinar la distribución de los datos se realizaron pruebas de normalidad Shapiro-Wilk y se realizó una comparación de medias con la prueba DSH (diferencia significativa honesta de Tukey) y un $\alpha=0.05$.

\section{RESUlTADOS}

Composición. En el muestreo del Bosque San Juan de Aragón se midieron 760 árboles agrupados en 10 familias botánicas; de las cuales Casuarinaceae, Proteaceae, Myrtaceae y Cupressaceae fueron las más frecuentes. Las angiospermas representaron el $82.49 \%$, y las gimnospermas el $17.50 \%$. La riqueza de especies fue de 12. Casuarina equisetifolia L., Eucalyptus camaldulensis Dhnh., Grevillea robusta A. Cunn. ex R. Br., Hesperocyparis lusitanica (Mill.) Bartel y Schinus molle L. conformaron el 76.25\% del total.

Variables dasométricas. El 31.7\% de los árboles presentaron diámetros de 7.6 a $15.1 \mathrm{~cm}$ (fig. 2A), y el $44.8 \%$ con alturas de 5.1 a $10 \mathrm{~m}$ (fig. 2B). Las diferencias entre especies fueron evidentes (cuadro 1). Eucalyptus y Casuarina, mostraron los mayores valores promedio de Dn (diámetro normal), At y Aba. Para Tamarix, los valores aumentaron debido a su carácter multifustal, que es una característica fenotípica de la especie. 

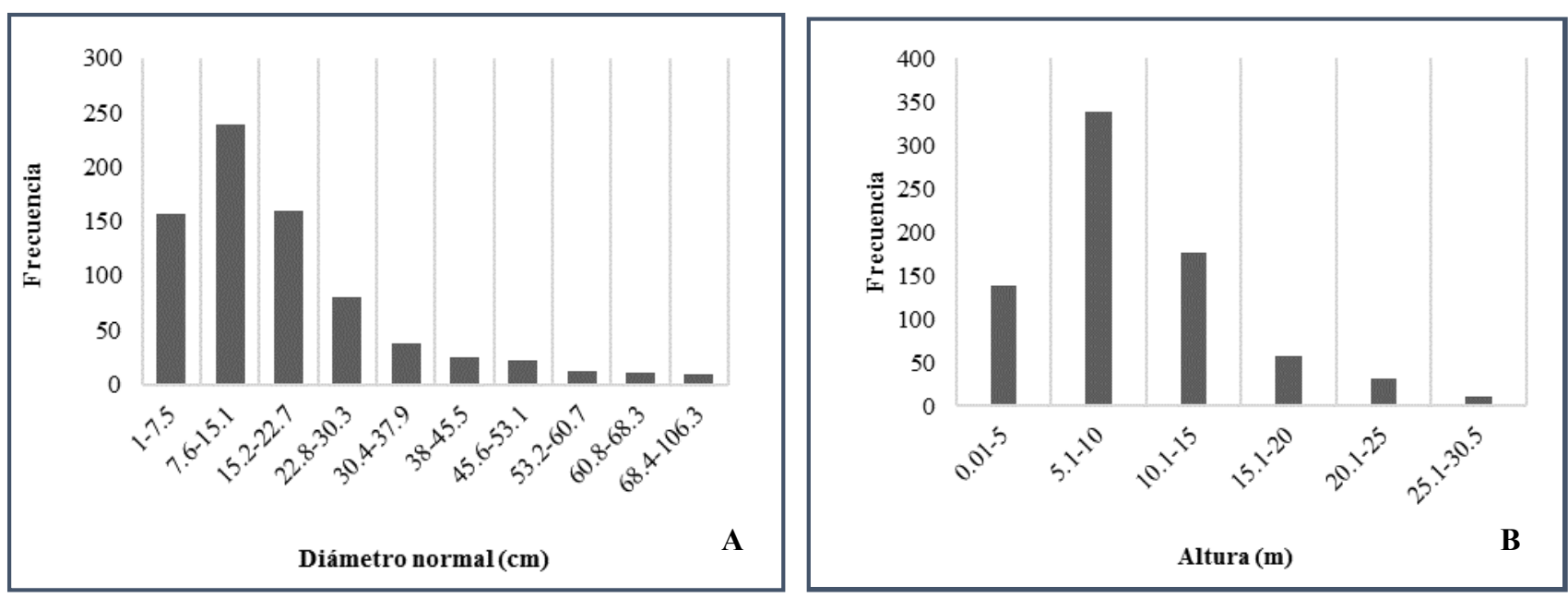

Fig. 2. Frecuencias para el diámetro normal (A) y la altura (B) de los árboles evaluados en el Bosque San Juan de Aragón, Ciudad de México.

Cuadro 1. Valores promedio de variables dasométricas por especie en el Bosque de San Juan de Aragón, Ciudad de México.

\begin{tabular}{lccc}
\hline \multirow{2}{*}{\multicolumn{1}{c}{ Especie }} & \multicolumn{3}{c}{ Variables $^{\star}$} \\
\cline { 2 - 4 } & $\begin{array}{c}\mathrm{Dn} \\
(\mathrm{cm})\end{array}$ & $\begin{array}{c}\text { At } \\
(\mathrm{m})\end{array}$ & $\begin{array}{c}\text { Aba } \\
\left(\mathrm{cm}^{2}\right)\end{array}$ \\
\hline Eucalyptus camaldulensis Dhnh. & $31.15^{\mathrm{a}}$ & $15.66^{\mathrm{a}}$ & $24.46^{\mathrm{a}}$ \\
Tamarix gallica L. & $28.58^{\mathrm{a}}$ & $11.38^{\mathrm{ab}}$ & $22.45^{\mathrm{a}}$ \\
Schinus molle L. & $26.62^{\mathrm{ab}}$ & $8.78 \mathrm{~b}^{\mathrm{cd}}$ & $20.92^{\mathrm{ab}}$ \\
Casuarina equisetifolia L. & $26.11^{\mathrm{ab}}$ & $13.28^{\mathrm{ab}}$ & $20.51^{\mathrm{ab}}$ \\
Acacia retinodes Schltdl. & $12.63^{\mathrm{bc}}$ & $5.98^{\mathrm{de}}$ & $9.92^{\mathrm{bc}}$ \\
Fraxinus uhdei (Wenz.) Lingelsh. & $10.38^{\mathrm{c}}$ & $5.87^{\mathrm{de}}$ & $8.15^{\mathrm{bc}}$ \\
Ligustrum lucidum Aiton & $12.76^{\mathrm{bc}}$ & $7.27^{\mathrm{cde}}$ & $10.02^{\mathrm{bc}}$ \\
Grevillea robusta A. Cunn. ex. R. Br. & $9.21^{\mathrm{c}}$ & $7.49^{\mathrm{cde}}$ & $7.23^{\mathrm{bc}}$ \\
Hesperocyparis lusitanica (Mill.) Bartel & $10.90^{\mathrm{c}}$ & $6.62 \mathrm{c}^{\mathrm{de}}$ & $8.56^{\mathrm{bc}}$ \\
Cupressus sempervirens L. & $6.43^{\mathrm{c}}$ & $4.15^{\mathrm{de}}$ & $5.05^{\mathrm{c}}$ \\
\hline
\end{tabular}

`Dn: diámetro normal; At: altura total; Aba: área basal. a, b, c, d: valores con la misma letra son significativamente iguales $(\mathrm{p} \leq 0.05)$.

Respecto a la densidad por especie, C. equisetifolia, G. robusta, H. lusitanica y E. camaldulensis presentaron valores de $23.60 \%, 17.24 \%, 13.66 \%$ y $12.06 \%$ del total, respectivamente.

Índices de diversidad. El índice de Simpson presentó un valor de 0.85, el cual indica una alta dominancia de especies y una baja riqueza; mientras que el índice de Berger-Parker corrobora lo anterior, ya que concentra los mayores valores de abundancia $(0.66)$ en cuatro de las 12 especies. La curva rango-abundancia por especie (fig. 3), muestra que Casuarina equisetifolia tuvo la mayor abundancia relativa dentro del bosque con 0.24 , seguida en orden decreciente por Grevillea robusta con 0.17 , Hesperocyparis lusitanica 0.14 y Eucalyptus camaldulensis con 0.11 , consideradas como las especies dominantes dentro del bosque. 


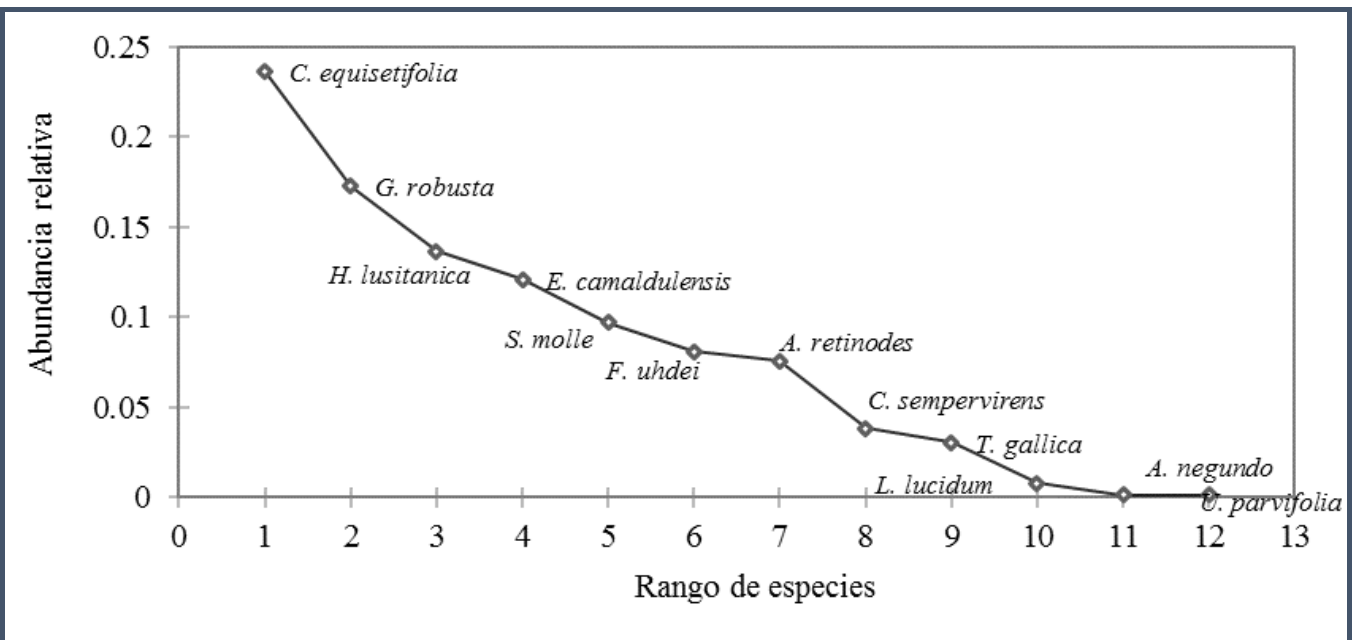

Fig. 3. Curva rango/abundancia por especie en el Bosque San Juan de Aragón, Ciudad de México.

En el índice de valor de importancia urbano se observaron tres patrones de acuerdo con McPherson \& Rowntree (1989). El primero, catalogado como dominante, fue para $C$. equisetifolia con el valor más alto (IVIU $=31.98)$, el segundo o codominante donde se ubicó a E. camaldulensis (IVIU $=19.96$ ) y el tercero o de débil dominancia, estuvo integrado por $S$. molle y $G$. robusta con valores de importancia muy similares (IVIU $=11.14$ y 11.63 , respectivamente). La diferencia porcentual entre estos grupos alcanzó el 10\% (cuadro 2).

Cuadro 2. Índices de valor de importancia urbano (IVIU) por especie arbórea en el Bosque de San Juan de Aragón, Ciudad de México.

\begin{tabular}{lcccccc}
\hline Especie & $\begin{array}{c}\text { Dominancia } \\
\text { relativa }\end{array}$ & $\begin{array}{c}\text { Frecuencia } \\
\text { relativa }\end{array}$ & $\begin{array}{c}\text { Altura } \\
\text { relativa }\end{array}$ & $\begin{array}{c}\text { Volumen } \\
\text { compuesto } \\
\text { relativo }\end{array}$ & $\begin{array}{c}\text { Área } \\
\text { superficial } \\
\text { relativa }\end{array}$ & $\begin{array}{c}\text { IVIU } \\
\text { \% }\end{array}$ \\
\hline C. equisetifolia & 33.045 & 23.64 & 32.71 & 34.46 & 36.03 & 31.98 \\
E. camaldulensis & 20.152 & 12.08 & 19.72 & 24.01 & 23.81 & 19.96 \\
S. molle & 14.042 & 9.69 & 8.96 & 12.03 & 10.96 & 11.14 \\
G. robusta & 8.514 & 17.26 & 13.48 & 9.18 & 9.71 & 11.63 \\
H. lusitanica & 7.983 & 13.68 & 9.44 & 6.68 & 6.63 & 8.88 \\
A. retinodes & 5.121 & 7.57 & 4.72 & 6.47 & 5.55 & 5.89 \\
T. gallica & 4.674 & 3.05 & 3.62 & 3.16 & 3.40 & 3.58 \\
F. uhdei & 4.504 & 8.10 & 4.95 & 3.29 & 3.18 & 4.81 \\
C. sempervirens & 1.327 & 3.85 & 1.67 & 0.39 & 0.41 & 1.53 \\
L. lucidum & 0.545 & 0.80 & 0.60 & 0.27 & 0.26 & 0.50 \\
A. negundo & 0.050 & 0.13 & 0.07 & 0.07 & 0.06 & 0.08 \\
U. parvifolia & 0.043 & 0.13 & 0.06 & 0.00 & 0.00 & 0.05 \\
\hline
\end{tabular}

Los altos valores del IVIU de C. equisetifolia y E. camaldulensis reflejan la importancia ecológica que ambas tienen dentro del BSJA, no sólo en términos de su dominancia, frecuencia y altura, sino también en cuanto al volumen de copa y área superficial. Especies con menor representación fueron $F$. uhdei y $C$. sempervirens, entre otras. 


\section{DISCUSIÓN}

La baja riqueza de especies del Bosque San Juan de Aragón, es comparable a la de otros bosques urbanos del mundo, y en algunos casos incluso menor. Jim \& Liu (2001), por ejemplo, reportan una riqueza de 215 especies arbóreas en 14 centros educativos con una superficie de 226 ha. En Bangalore, India, Nagendra \& Gopal (2010), identificaron sólo seis especies en 24 ha de arbolado de alineación, valores que sin duda son menores a los registrados en el BSJA donde se identificaron 12 especies. Un caso excepcional, fue publicado por Muthulingam \& Thangavel (2012), que concluyen la presencia de 45 especies, 42 géneros y 21 familias en espacios verdes institucionales del Distrito de Chennai, China en tan sólo una hectárea de terreno, sin embargo, casos como este son poco comunes. Un carácter adicional al valor de riqueza del BSJA, es la alta dominancia de géneros, especies y familias botánicas. Bajo la premisa de protección y conservación de bosques urbanos contra plagas y enfermedades exóticas y/o nativas, Santamour (1990), planteó la regla 10-20-30, y en torno a esta propuesta, se asume que en términos de diversidad arbórea, un bosque urbano se considera adecuado, cuando la presencia de una especie arbórea no excede el 10\%, respecto a un género no más del $20 \%$ y no más del $30 \%$ de una familia (Kendal, Dobbs, \& Lohr, 2014). En el BSJA, al menos cuatro especies, Casuarina equisetifolia, Grevillea robusta, Hesperocyparis lusitanica y Eucalyptus camaldulensis mostraron una frecuencia mayor al $10 \%$ respecto a la especie, Casuarina rebasó el $20 \%$ por género, y ninguna familia superó el $30 \%$ establecido por Santamour (1990). Esta uniformidad taxonómica ocurre también en otras áreas verdes urbanas del mundo, caso concreto el de Flanders, Bélgica, donde el análisis de diversidad arbórea en 15 parques reveló que cuatro angiospermas representan la mayor cobertura, entre estas: Fagus sylvatica L., Acer pseudoplatanus L., Quercus robur L., y Fraxinus excelsior (Cornelis \& Hermy, 2004), mientras que en la ciudad de Guangzhou, China, se reportan a Ficus virens Aiton, Maleleuca leucandendra L. y una palmera, Caryota mitis Loureiro (Jim \& Liu, 2001) con las mayores frecuencias.

Debido al incremento de plagas y enfermedades exóticas con efectos devastadores (Alvey, 2006), es posible reducir la vulnerabilidad del bosque urbano incrementando su diversidad biológica y genética, de ser posible (Kendal et al., 2014). La ausencia de barreras biológicas y especies resistentes contra agentes de estrés y muerte, podrían desencadenar problemas fitosanitarios graves en el BSJA o áreas adyacentes (Zoológico de Aragón), o en cualquier parte de la Ciudad de México. La devastación de muchos bosques urbanos del mundo a causa de distintos agentes de daño, se ha ilustrado en diversos estudios, por ejemplo, el hongo Ophiostoma ulmi (Buisman) Menlin \& Nannf., causante de la enfermedad del olmo holandés, diezmó las poblaciones de Ulmus americana en Estados Unidos de Norte América y Canadá (Tainter \& Baker, 1996). Un caso similar, y que ha provocado la muerte de millones de fresnos, es el barrenador esmeralda (Agrilus planipennis Fairmaire); investigaciones recientes indican que las especies más afectadas son Fraxinus pennsylvanica Marsh. F. americana L., $F$. nigra Marsh, y F. quadrangulata Michx (Kendal et al., 2014; States Department of Agriculture, 2015). Estos hechos y muchos otros evidencian la importancia de incrementar la diversidad de especies arbóreas en los centros urbanos, sin dejar de lado los estudios de capacidad adaptativa por especie, preferencias edafo-climáticas, ubicación geográfica y sin duda, de un manejo adecuado (Wania, Kühn, \& Klotz, 2006; Jim \& Liu, 2001).

Las especies no nativas conforman un alto porcentaje de las especies arbóreas dentro de las áreas urbanas, fenómeno que algunos consideran saludable, pues es una manera fácil y rápida de incrementar la diversidad (McKinney, 2006), sin embargo, no toman en cuenta la problemática que a largo plazo puede significar la introducción de estas especies en zonas con distintas condiciones ambientales y que a futuro generarán un manejo intensivo. En la Ciudad de México, no se sabe con certeza cuántas, ni cuáles especies arbóreas son nativas, pero en lo que al BSJA se refiere, la riqueza de especies está en función de especies no nativas $(C$. equisetifolia, E. camaldulensis, G. robusta y A. retinodes), las cuales en apariencia se han adaptado a este sitio, no por ello son la mejor opción. 
La homogeneidad de géneros y especies es poco recomendable en términos de salud (Li et al., 2011; Yang, Zhou, Ke, \& Xiao, 2012), aunque bajo la perspectiva de manejo podría ser útil. La presencia de pocas especies dominantes podría tener un menor costo de mantenimiento debido a que las actividades de manejo serían repetitivas, lo que representaría una ventaja económica y menor desgaste humano (McPherson \& Rowntree, 1989).

En general, los árboles del BSJA, presentaron porte pequeño, con excepción de algunos individuos grandes de eucalipto y casuarina. Los continuos programas de reposición e introducción de nuevo arbolado podrían estar incrementando la plantación de árboles jóvenes con $\mathrm{Dn}<20 \mathrm{~cm}$ y altura menor a $15 \mathrm{~m}$. Árboles grandes pueden proveer mayores servicios ecosistémicos (mayor remoción de contaminantes y altas tasas de carbono secuestrado y almacenado), comparado con árboles de menor tamaño (Baró et al., 2014). En lo que respecta al BSJA, sólo el $10.12 \%$ de los árboles tienen diámetros $>30 \mathrm{~cm}$. La carencia de árboles grandes son posiblemente, reflejo de acciones de manejo pasadas (Cumming, Galvin, Rabaglia, Cumming, \& Twardus, 2001). Teniendo como base el diámetro normal, una distribución aceptable de diámetros en un bosque urbano deberá incluir las siguientes categorías: $40 \%<20$ $\mathrm{cm}, 30 \%$ entre $20-40 \mathrm{~cm}, 20 \%$ de $40-60 \mathrm{~cm}$ y $10 \%$ mayor a $60 \mathrm{~cm}$. De acuerdo con los estudios de Richards (1983), los bosques urbanos con esta distribución de diámetros se pueden considerar estables, y a futuro, la alta frecuencia de árboles jóvenes, puede ser benéfico pues a mediano plazo estos podrán reemplazar a individuos maduros o a aquellos que pudieran declinar ante la presencia de plagas y enfermedades (McPherson \& Rowntree, 1989), de hecho, este último estudio es uno de los más completos sobre la diversidad arbórea en 22 estados de la Unión Americana.

En lo que respecta a los índices de diversidad evaluados (Simpson y Berger-Parker) éstos reflejan que en el BSJA existe una baja riqueza y alta dominancia de pocas especies, por ello, es recomendable establecer programas que incidan en la mejora de estos aspectos (Landeros \& Cerna, 2007). El panorama general es que unas pocas especies constituyen una gran proporción de las comunidades arbóreas en los centros urbanos. En Chicago, EU, por ejemplo, cuatro de las especies comprenden dos terceras partes de su arbolado (Li et al., 2011) y en México, el mismo número de especies constituyen el $49 \%$ de las comunidades arbóreas e incluso algunas de ellas están catalogadas como monumentos urbanísticos, entre ellas, Fraxinus uhdei (MezaAguilar, 2015).

Un reto en el estudio de la estructura y diversidad arbórea es la generación de índices ecológicos que representen la importancia de las distintas especies arbóreas en la ciudad e identificar posibles patrones y tendencias, especialmente en investigaciones a largo plazo (McPherson \& Rowntree, 1989). En este estudio, con base en los altos valores de dominancia (área basal), frecuencia y dimensiones (mayor altura, área superficial y volumen compuesto de copa), las especies C. equisetifolia, E. camaldulensis y G. robusta son de gran importancia ecológica dentro del BSJA al poseer los mayores IVIU. Es la primera vez que se utilizan variables tridimensionales para calcular este índice el cual no sólo aporta una gran cantidad de información en poco tiempo, sino también proporciona el valor ecológico de cada especie en el área de estudio aportando elementos cuantitativos como cantidad de biomasa y que refiere Lozada-Dávila (2010). Estudios similares al presente, emplean otras variables ecológicas para determinar valores de importancia, por ejemplo, densidad, frecuencia y área basal (Burton, Samuelson, \& Pan, 2005). Esto hace patente, la versatilidad de calcular índices de valor de importancia específicos para cada área, objetivos de estudio y la función que desempeña el área verde dentro de las ciudades.

\section{CONCLUSIONES}

El uso de la diversidad y estructura como un indicador de salud en el escenario del Bosque San Juan de Aragón en la Ciudad de México, mostró que la riqueza de especies es equiparable a 
distintos bosques urbanos, destacando la dominancia y frecuencia de especies arbóreas no nativas (casuarinas, eucaliptos, acacias, y grevileas), lo cual no es recomendable desde la perspectiva de salud.

Se encontró una alta frecuencia de árboles de diámetros pequeños, los cuales conforman una reserva de biomasa que a futuro podrá sustituir individuos enfermos, muertos o en proceso de declinación.

Se determinó por primera vez un índice de valor de importancia urbano (IVIU), conformado por variables absolutas y compuestas de copa que permiten jerarquizar la importancia de cada especie de manera horizontal al incluir parámetros ecológicos como la dominancia y frecuencia, así como de manera vertical y tridimensional, al considerar la altura, el volumen y el área superficial de copa. Finalmente, su aplicación en el ambiente urbano, contribuye a la identificación de las especies arbóreas con altos niveles de biomasa y candidatos para proveer de mayores beneficios a la población.

\section{Agradecimientos}

A los líderes de los Indicadores de Salud del Servicio Forestal de Estados Unidos de América del Norte, (USDA FS), especialmente a la doctora Bethany Schulz.

\section{LITERATURA CITADA}

Alvey, A. A. (2006). Promoting and preserving biodiversity in the urban forest. Urban Forestry and Urban Greening, 5(4), 195-201. https://doi.org/10.1016/j.ufug.2006.09.003

Baró, F., Chaparro, L., Gómez-Baggethun, E., Langemeyer, J., Nowak, D. J., \& Terradas, J. (2014). Contribution of ecosystem services to air quality and climate change mitigation policies: The case of urban forests in Barcelona, Spain. Ambio, 43(4), 466-479. https://doi.org/10.1007/s13280-014-0507-x

Beckett, K. P., Freer-Smith, P. H., \& Taylor, G. (2000). Effective tree species for local air quality management. Journal of Arboriculture, 26(1), 12-19.

Buckelew, C. A., Twardus, D. B., \& Nowak, D. J. (2008). Urban Forest Health Monitoring: Large-scale assessments in the United States. Arboriculture \& Urban Forestry, 34(6), 341-346.

Burkhart, H. E., \& Tomé, M. (2012). Modeling Forest Trees and Stands. Dordrecht: Springer Netherlands. https://doi.org/10.1007/978-90-481-3170-9

Burton, M. L., Samuelson, L. J., \& Pan, S. (2005). Riparian woody plant diversity and forest structure along an urban-rural gradient. Urban Ecosystems, 8(1), 93-106. https://doi.org/10.1007/s11252-005-1421-6

Cornelis, J., \& Hermy, M. (2004). Biodiversity relationships in urban and suburban parks in Flanders. Landscape and Urban Planning, 69(4), 385-401. https://doi.org/10.1016/j.landurbplan.2003.10.038

Cumming, a. B., Galvin, M. F., Rabaglia, R. J., Cumming, J. R., \& Twardus, D. B. (2001). Forest health monitoring protocols applied to roadside trees in Maryland. Journal of Arboriculture, 27(3), 126-138.

Flores-Xolocotzi, R., \& González-Guillén, M. (2010). Planificación de sistemas de áreas verdes y parques públicos. Revista Mexicana de Ciencias Forestales, 1(1), 17-24.

Galvin, M. F. (1995). a Methodology for Assessing and Managing Biodiversity in Street Tree Populations : a Case Study, (July), 124-128.

García-Mayoral, L. E., Valdez-Hernández, J. I., Luna-Cavazos, M., \& López-Morgado, R. (2015). Estructura y diversidad arbórea en sistemas agroforestales de café en la Sierra de Atoyac, Veracruz. Madera y Bosques, 21(3), 69-82.

Janhäll, S. (2015). Review on urban vegetation and particle air pollution e Deposition and 
dispersion. Atmospheric Environment, 105, 130-137. https://doi.org/10.1016/ j.atmosenv.2015.01.052

Jim, C. ., \& Liu, H. . (2001). Species diversity of three major urban forest types in Guangzhou City, China. Forest Ecology and Management, 146(1), 99-114. https://doi.org/10.1016/S0378-1127(00)00449-7

Kendal, D., Dobbs, C., \& Lohr, V. I. (2014). Global patterns of diversity in the urban forest: Is there evidence to support the 10/20/30 rule? Urban Forestry and Urban Greening, 13(3), 411-417. https://doi.org/10.1016/j.ufug.2014.04.004

Korhonen, L., Vauhkonen, J., Virolainen, A., Hovi, A., \& Korpela, I. (2013). International Journal of Remote Sensing Estimation of tree crown volume from airborne lidar data using computational geometry Estimation of tree crown volume from airborne lidar data using computational geometry. International Journal of Remote Sensing, 34(20), 7236-7248. https://doi.org/10.1080/01431161.2013.817715

Landeros, J., \& Cerna, E. (2007). Patrones de asociación de especies y sustentabilidad (Species association patterns and sustainability). International Journal of Good Conscience. Octubre, 3(1), 632-660.

Li, Y. Y., Wang, X. R., \& Huang, C. L. (2011). Key street tree species selection in urban areas. African Journal of Agricultural Research, 6(15), 3539-3550. https://doi.org/10.5897/AJAR11.461

Lozada-Dávila, R. (2010). Consideraciones metodológicas sobre los estudios de comunidades forestales Methodological considerations about studies of forest communities. Revista Forestal Venezolana, 54(1), 77-88.

Magurran, A. E. (2004). Magurran 2004.pdf. Measuring Biological Diversity.

McKinney, M. L. (2006). Urbanization as a major cause of biotic homogenization. Biological Conservation, 127(3), 247-260. https://doi.org/10.1016/j.biocon.2005.09.005

McPherson, G., \& Rowntree, R. A. (1989). Using Structural Measures to Compare twenty-two U.S. street tree population. Landscape Journal, 9(1), 13-23. https://doi.org/10.3368/1j.8.1.13

Meza Aguilar, M. del C. (2015). The trees of Mexico city: guardians of their image and of the environment. Bitácora Arquitectura, (31), 96-103.

Mori, J., Hanslin, H. M., Burchi, G., \& Sæbø, A. (2015). Particulate matter and element accumulation on coniferous trees at different distances from a highway. Urban Forestry and Urban Greening, 14(1), 170-177. https://doi.org/10.1016/j.ufug.2014.09.005

Muelle-Dombois, D., \& Ellenberg, H. (1974). Aims \& Methods of vegetation ecology. New York: John Wiley \& Sons.

Muthulingam, U., \& Thangavel, S. (2012). Density, diversity and richness of woody plants in urban green spaces: A case study in Chennai metropolitan city. Urban Forestry and Urban Greening, 11(4), 450-459. https://doi.org/10.1016/j.ufug.2012.08.003

Nagendra, H., \& Gopal, D. (2010). Street trees in Bangalore: Density, diversity, composition and distribution. Urban Forestry and Urban Greening, 9(2), 129-137. https://doi.org/10.1016/j.ufug.2009.12.005

Nowak, D. J., \& Heisler, G. M. (2010). Air Quality Effects of Urban Trees and Parks.

Potter, K. M. (2017). Introduction. In K. M. Potter \& B. L. Conkling (Eds.), Forest Health Monitoring: National Status, Trends, and Analysis 2016 (p. 195). Asheville, NC.

Randolph, K. C., Morin, R. S., \& Steinman, J. (2010). Descriptive Statistics of Tree Crown Condition in the North Central United States. Ashville NC.

Räsänen, J. V., Holopainen, T., Joutsensaari, J., Ndam, C., Pasanen, P., Rinnan, A., \& Kivimäenpää, M. (2013). Effects of species-specific leaf characteristics and reduced water availability on fine particle capture efficiency of trees. Environmental Pollution, 183, 64-70. https://doi.org/10.1016/j.envpol.2013.05.015

Richards, N. A. (1983). Diversity and stability in a street tree population. Urban Ecology, 7(2), 159-171. https://doi.org/10.1016/0304-4009(83)90034-7

Romhan-de la Vega, C. F., \& Ramírez-Maldonado, H. (2010). Dendrometría. México: Universidad Autónoma Chapingo. 
Recibido:

20/abril/2018

Aceptado:

6/diciembre/2018
Saavedra-Romero, L. L., Alvarado-Rosales, D., Hernández-De La Rosa, P., Martínez-Trinidad, T., Mora-Aguilera, G., \& Villa-Castillo, J. (2016). Crown condition, a health indicator in urban trees of the San Juan de Aragon Park, Mexico City. Madera y Bosques, 22(2), $15-27$.

Santamour, F. S. (1990). Trees for urban planting: Diversity, Uniformity, and Common Sense. Proceedings of the Seventh Conference of The Metropolitan Tree Improvement Alliance, 7, 57-65.

Savard, J. P. L., Clergeau, P., \& Mennechez, G. (2000). Biodiversity concepts and urban ecosystems. Landscape and Urban Planning, 48(3-4), 131-142. https://doi.org/10.1016/S0169-2046(00)00037-2

Schomaker, M. E., Zarnoch, S. J., Bechtold, W. A., Latele, D. J., Burkman, W. G., \& Cox, S. M. (2007). Crown-Condition Classification: A Guide to Data Collection and Analysis. USDA Gen. Tech. Rep. SRS-102. U.S. Department of Agriculture Forest Service, Asheville, NC., 92.

Schroeder, T. A., Healey, S. P., \& Moisen, G. G. (2010). Evaluating the compatibility of american and mexican national forest inventory data. In 2010 Joint Meeting of the Forest Inventory and Analysis (FIA) Symposium and the Southern Measurationists (pp. 15-22).

Schulz, B. K. (2017). Changes on vegetation indicators plots: small samples reveals significant trends. In K. M. Potter \& B. L. Conkling (Eds.), Forest Health Monitoring: National Status, Trends, and Analysis 2016 (p. 195). Asheville, NC.: U.S. Department of Agriculture, Forest Service.

Schulz, B. K., Bechtold, W. a, \& Zarnoch, S. J. (2009). Sampling and estimation procedures for the vegetation diversity and structure indicator. U S Forest Service Pacific Northwest Research Station General Technical Report PNW-GTR, 1-53.

Tainter, F. H., \& Baker, F. A. (1996). Principles of forest pathology. John Wiley.

Tellez-Garcia, C. P., \& Valdez-Hernandez, J. I. (2012). Caracterizacion estructural del manglar en el estero palo verde, laguna de cuyutlan, Colima. Revista Chapingo, Serie Ciencias Forestales y Del Ambiente, 18(3), 395-408. https://doi.org/10.5154/r.rchscfa.2010.11.101

Troxel, B., Piana, M., Ashton, M. S., \& Murphy-Dunning, C. (2013). Relationships between bole and crown size for young urban trees in the northeastern USA. Urban Forestry \& Urban Greening. https://doi.org/10.1016/j.ufug.2013.02.006

United States Department of Agriculture, U. (2015). Pest Alert: Barrenador esmeralda del fresno.

Woodall, C. W., Amacher, M. C., Bechtold, W. A., Coulston, J. W., Jovan, S., Perry, H. C., ... Will-Wolf, S. (2011). Status and future of the forest health indicators program of the USA Field data from most sample years and indicators. Environ Monit Assess, 177, 419-436. https://doi.org/10.1007/s10661-010-1644-8

Yang, J., Zhou, J., Ke, Y., \& Xiao, J. (2012). Assessing the structure and stability of street trees in Lhasa, China. Urban Forestry and Urban Greening, 11(4), 432-438. https://doi.org/10.1016/j.ufug.2012.07.002

Zaragoza-Hernández, A. Y., Cetina-Alcalá, V. M., López-López, M. A., Chacalo-Hilú, A., De la Isla, M. L., Alvarado-Rosales, D., \& González-Rosas, H. (2015). Identification of tree damages of three parks of Mexico City. Revista Mexicana de Ciencias Forestales, 6(32), 63-82.

Zarnoch, S. J., Bechtold, W. a, \& Stolte, K. W. (2004). Using crown condition variables as indicators of forest health. Canadian Journal of Forest Research, 34(5), 1057-1070. https://doi.org/10.1139/x03-277. 